\title{
Grand Challenges in Soft Matter
}

\author{
Raffaele Mezzenga ${ }^{1,2 *}$ \\ ${ }^{1}$ Department of Health Sciences and Technology, ETH Zurich, Zurich, Switzerland, ${ }^{2}$ Department of Materials, ETH Zurich, Zurich, \\ Switzerland
}

Keywords: polymers, gels, liquid crystals, self-assembly, colloids and emulsions, granular matter, foams, biological soft matter

\section{INTRODUCTION}

From the food we cook in the kitchen, to the living cells which form our bodies, Soft Matter is ubiquitous in our every-day lives. How well do we understand Soft Matter and what do we miss to improve our comprehension of this fascinating class of matter? What are the challenges we face to translate fundamental understanding into impactful applications? This short personal perspective tackles some of the challenges associated with Soft Matter and provides possible directions forwards in addressing these challenges.

From a chemistry perspective, the field of Soft Matter is just about a century-old, as one could very well consider the 1920 seminal paper of Staudinger "Über Polymerisation," (Staudinger, 1920) as the spark which set the fire to the explosion of polymer chemistry, one of the core pillars of Soft Matter today. Since then, polymer chemistry has continuously reshaped the landscape of polymers. Polymers are easy to produce and can be cast or moulded into any possible shape; polymer synthesis can be engineered by catalysts to have very low-energy requirements and deliver polymers with high control precision in their molecular architecture. It is for these reasons (and much more) that today "plastics" has become one of the most widely spread man-made materials around the globe, but it is also via advanced polymer chemistry approaches that we are today actively seeking valid solutions for switching from petroleum-based plastics to biodegradable polymers (Tian et al., 2012), to enter into a more sustainable era of polymers, in full harmony with the environment and reducing their global impact on our society.

From a physics perspective, the field of Soft Matter as a distinct scientific discipline effectively started only 15 years earlier, with the 1905 annus mirabilis seminal paper of Albert Einstein on Brownian motion (Einstein, 1905). This paper introduces a few ground-breaking concepts over which Soft Matter is still centred today, such as for example the linear $t$ dependence of the mean square displacement of colloidal particles, or the derivation of the expression for the diffusion coefficient of a colloidal particle, an equation to which we today refer by as the Stokes-Einstein law.

But perhaps one sentence is particularly revealing in that paper by Einstein: "... and is not apparent why a number of suspended particles should not produce the same osmotic pressure as the same number of molecules" (Einstein et al., 1956). The underlying assumption behind this sentence is that, by being the particles "suspended" they must possess an energy of the order of $\mathrm{K}_{\mathrm{b}} \mathrm{T}$ : if not they would either sediment to the bottom or float to the surface of the fluid on which they are suspended. One could actually start from this very same sentence to provide an accurate definition of a colloidal particle, by defining it as any entity with a kinetic energy of the order of $\mathrm{K}_{\mathrm{b}} \mathrm{T}$, or whose trajectory follows a random walk, for which its mean square displacement acquires a time dependence linear with time t. One is then confronted with a possible first definition of Soft Matter as any material, may this be a liquid, a fluid, a glass or solid, organized by the assembly of building blocks whose energy is on the order of a few $\mathrm{K}_{\mathrm{b}} \mathrm{T}$.

Taking one step further the sentence of Einstein quoted above, let consider a classical particle of radius $\mathrm{R}$ and density $\rho_{S}$ moving in a fluid of density $\rho_{L}$ and viscosity $\eta$ having reached a terminal steady-state velocity $v$ based on the equilibrium between the forces of gravity $\left(4 / 3 \pi \rho_{S} g R^{3}\right)$, buoyancy 
$\left(-4 / 3 \pi \rho_{L} g R^{3}\right)$, and the Stokes viscous friction $(-6 \pi \eta R v)$. By equating these contributions, one finds that $v=2\left(\rho_{S-} \rho_{L}\right) g R^{2} / 9 \eta$. As just discussed, this particle will then become a colloidal particle when $2 / 3 \pi \rho_{S} g R^{3} v^{2}$, its kinetic energy, becomes equal to $1 / 2 K_{B} T$, as required by the law of equipartition of energy. By using the value of the limiting velocity derived few lines above and equating the two energies, this ultimately implies that a colloidal object must have a size:

$$
R \leq \frac{3^{5 / 7}\left(K_{B} T\right)^{1 / 7} \eta^{2 / 7}}{2^{4 / 7} \pi^{1 / 7} g^{2 / 7} \rho_{S}^{1 / 7}\left(\rho_{S}-\rho_{L}\right)^{2 / 7}}
$$

Equation 1 provides a rationale for defining the limiting colloidal size as a function of the physical characteristics of the particle and the medium on which it is suspended. For example, for polystyrene colloidal particles suspended in water at room temperature, Eq. (1) sets a limit to $R \leq 13 \mu m$. But what happens if $R$ is actually larger than the limit sets by Eq. (1)? Note that Eq. (1) does not distinguish between a large air bubble in a liquid foam floating up to the surface, where the term $\left(\rho_{S}-\right.$ $\rho_{L}$ ) is negative, or a grain of sand from a sediment in a river's delta, where the term $\left(\rho_{S}-\rho_{L}\right)$ is positive, since this term is first squared and then further elevated to $1 / 7$ in Eq. (1). In such a range of length scales, we are still in the field of Soft Matter, but the object is simply no longer colloidal. We then enter the realm of granular media for which stochastic approaches do no longer apply to describe a trajectory of a particle, and a deterministic description becomes more appropriate. Yet, the challenge associated with granular matter, a vibrant area of modern Soft Matter, is that universal constitutive equations do not yet exist, as recently highlighted in two other Frontiers Field Challenge perspectives (Hansen, 2014; van der Gucht, 2018), and continuum phenomenological models are more successful in describing experimental evidence.

Whether you look at Soft Matter from a chemistry or physicsoriented perspective, one very important overarching aspect immediately emerges in this field of science: interdisciplinarity. Today Soft Matter is composed of a multitude of sub-fields deeply rooted into other consolidated disciplines such as physics, chemistry, biology among the fundamental fields and material science, engineering and nanotechnology among the more applied ones.

One example which highlights particularly well how interdisciplinarity becomes the dominating trait in Soft Matter is the phenomenon of liquid-liquid phase separation (LLPS) of macromolecular solutions, which is currently at the forefront of biological soft matter (Dolgin, 2018). LLPS in the context of biology is indeed critically important in many different phenomena, such as the formation of cellular DNA-rich nucleoli in the nucleoplasm (Brangwynne et al., 2011) and RNA-rich membraneless organelles in the cytoplasm, among which P granules (Brangwynne et al., 2009), to cite only a couple of examples.

Generally speaking, liquid-liquid phase separation in a polymer solution is the process of de-mixing, most commonly, polymer-rich droplets from a continuous polymer-depleted solution. As the system evolves due to changes in the solvent quality, the droplets and the continuous phase follow two opposite branches of a binodal line describing their composition evolutions. By the early ' 50 s Flory had already set the basis for understanding this process by separately calculating the contributions of enthalpy and entropy in a polymer solution based on classical statistical mechanics concepts (Flory, 1953). The famous Flory-Huggins equation has since then become a cornerstone of polymer thermodynamics and has proven useful in a countless number of occasions. Yet, when it comes to biological liquid-liquid phase separation, the process becomes soon massively more complicated: to start the process often involves not only two components (the polymer and the solvent), but three components such as water, a protein and either DNA or RNA; secondly, these compounds are polyelectrolytic in nature with opposite charges, and, as for proteins, they are polyampholytes, i.e. polyelectrolytes with both positive and negative charges (and a non-homogeneous linear charge distribution), leading to electrostatic complexes called coacervates; third, the presence of specific ion interactions make electrostatic significantly more complex than current polyelectrolyte theories can handle; fourth and last, water is a massively more complex solvent than any other organic solvent. With such a complicated scenario in mind, translation of concepts based on statistical physics as the Flory-Huggins equation, albeit very general, becomes non-trivial to be applied to biological LLPS. Although attempts to include electrostaticinteractions in the enthalpic term of the Flory-Huggins started soon after the seminal work of Flory (Overbeek and Voorn, 1957), the complexity imposed by biology is such to call still today for new major adjustments (Brangwynne et al., 2015), within and beyond the mean-field treatment introduced by Flory, which contribute to a vibrant part of current biological Soft Matter.

As opposed to liquid-liquid phase separation, where the process is a trade-off between enthalpy (favouring de-mixing) and entropy (favouring mixing), Liquid-Liquid Crystalline Phase Separation (LLCPS) is a fundamentally distinct phenomenon where spontaneous phase separation occurs exclusively based on entropic reasons (this time favouring de-mixing) whenever rigid filamentous colloids with a high excluded volume exists in suspension. The fundamental understanding of the physics of anisotropic colloids is rooted in the seminal 1949 Osanger paper (Onsager, 1949), in which a robust thermodynamic theory is introduced setting the starting point to any possible approach to anisotropic colloidal systems. Yet, the understanding of the dynamics by which LLCPS evolves via nucleation and growth, emerging from the trade-off of thermodynamics and kinetics is, surprisingly, still on its infancy and only recently theoretical descriptions capable to map experimental observations start to surface (Azzari et al., 2021). It is to be auspicated that this field will develop rapidly as LLCPS may have tremendous implications in biology, for example, where filamentous colloids such as amyloid fibrils formed by aggregation of pathological proteins are involved in several devastating neurodegenerative diseases, which could very well stem from LLCPS itself. An improved understanding from a Soft Matter perspective of these phenomena could then have direct implications in other important fields such as biology and medicine. 
On the synthetic side, the progresses in several branches of chemistry have made the library of molecules available to Soft Matter scientists virtually unlimited. Organic and inorganic chemistry have been enriched by the more recent supramolecular chemistry to bring molecular design to a different level (Lehn, 1993). This enables, among many other things, tuning self-assembly, molecular recognition, stimuliresponsive aggregation and dissolution of molecular aggregates into a highly controllable process where the design of functional materials becomes possible well beyond what was on the menu only one or 2 decades ago (Stupp and Palmer, 2014). All these tools make Soft Matter a field in which it becomes possible not only better understanding the world around us, but also to design, engineer and build from scratch new materials available to next generations.

It is truly impossible to do justice to Soft Matter diversity, interdisciplinarity and richness in such a short perspective, and I have therefore touched only on a very few highly personal topics with which I am more familiar, or which are closer to my heart. In

\section{REFERENCES}

Azzari, P., Bagnani, M., and Mezzenga, R. (2021). Liquid-Liquid Crystalline Phase Separation in Biological Filamentous Colloids: Nucleation, Growth and OrderOrder Transitions of Cholesteric Tactoids. Soft Matter 17, 6627-6636. doi:10.1039/d1sm00466b

Brangwynne, C. P., Eckmann, C. R., Courson, D. S., Rybarska, A., Hoege, C., Gharakhani, J., et al. (2009). Germline P Granules Are Liquid Droplets that Localize by Controlled Dissolution/condensation. Science 324, 1729-1732. doi:10.1126/science.1172046

Brangwynne, C. P., Mitchison, T. J., and Hyman, A. A. (2011). Active Liquid-like Behavior of Nucleoli Determines Their Size and Shape in Xenopus laevis Oocytes. Proc. Natl. Acad. Sci. 108, 4334-4339. doi:10.1073/pnas.1017150108

Brangwynne, C. P., Tompa, P., and Pappu, R. V. (2015). Polymer Physics of Intracellular Phase Transitions. Nat. Phys 11, 899-904. doi:10.1038/nphys3532

Dolgin, E. (2018). What Lava Lamps and Vinaigrette Can Teach Us about Cell Biology. Nature 555, 300-302. doi:10.1038/d41586-018-03070-2

Einstein, A. (1956). "English Translation by Furth \& Cowper," in Investigations on the Theory of the Brownian Movement. Editors R. Fürth and A. D. Cowper (Dover Publications).

Einstein, A. (1905). Über die von der molekularkinetischen Theorie der Wärme geforderte Bewegung von in ruhenden Flüssigkeiten suspendierten Teilchen. Ann. Phys. 322, 549-560. doi:10.1002/andp.19053220806

Flory, P. J. (1953). Principles of Polymer Chemistry. Ithaca, New York: Cornell University Press.

Hansen, A. (2014). Grand Challenges in Interdisciplinary Physics. Front. Phys. 2, 58. doi:10.3389/fphy.2014.00058

Lehn, J.-M. (1993). Supramolecular Chemistry. Science 260, 1762-1763. doi:10.1126/science. 8511582

Onsager, L. (1949). The Effects of Shape on the Interaction of Colloidal Particles. Ann. NY Acad. Sci. 51, 627-659. doi:10.1111/j.1749-6632.1949.tb27296.x particular, in this Field Grand Challenge (very personal) opinion about Soft Matter, I have deliberately taken the approach of starting from milestones set by giants in the field such as Staudinger, Einstein, Flory, Onsager and others, to highlight how it is possible, by learning from past lessons, to translate seminal concepts with renewed perspectives and momentum into new and future challenges surfacing every day in Soft Matter. I am convinced that it is first by digging into past experiences that future challenges can best be addressed. In this respect, Soft Matter is providing a unique, inspiring, exciting and vibrating playground on which scientists, technologists and engineers can meet to shape, together, a bright future.

\section{AUTHOR CONTRIBUTIONS}

The author confirms being the sole contributor of this work and has approved it for publication.

Overbeek, J. T. G., and Voorn, M. J. (1957). Phase Separation in Polyelectrolyte Solutions. Theory of Complex Coacervation. J. Cell. Comp. Physiol. 49, 7-26. doi:10.1002/jcp.1030490404

Staudinger, H. (1920). Über Polymerisation. Ber. Dtsch. Chem. Ges. A/b 53 , 1073-1085. doi:10.1002/cber.19200530627

Stupp, S. I., and Palmer, L. C. (2014). Supramolecular Chemistry and SelfAssembly in Organic Materials Design. Chem. Mater. 26, 507-518. doi: $10.1021 / \mathrm{cm} 403028 \mathrm{~b}$

Tian, H., Tang, Z., Zhuang, X., Chen, X., and Jing, X. (2012). Biodegradable Synthetic Polymers: Preparation, Functionalization and Biomedical Application. Prog. Polym. Sci. 37, 237-280. doi:10.1016/ j.progpolymsci.2011.06.004

van der Gucht, J. (2018). Grand Challenges in Soft Matter Physics. Front. Phys. 6 , 87. doi:10.3389/fphy. 2018.00087

Conflict of Interest: The author declares that the research was conducted in the absence of any commercial or financial relationships that could be construed as a potential conflict of interest.

Publisher's Note: All claims expressed in this article are solely those of the authors and do not necessarily represent those of their affiliated organizations, or those of the publisher, the editors and the reviewers. Any product that may be evaluated in this article, or claim that may be made by its manufacturer, is not guaranteed or endorsed by the publisher.

Copyright () 2021 Mezzenga. This is an open-access article distributed under the terms of the Creative Commons Attribution License (CC BY). The use, distribution or reproduction in other forums is permitted, provided the original author(s) and the copyright owner(s) are credited and that the original publication in this journal is cited, in accordance with accepted academic practice. No use, distribution or reproduction is permitted which does not comply with these terms. 